\title{
Uma Introdução ao Processo de Nascimento e Assassinato
}

Adriano Gonçalves Lima

\author{
DisSERTAÇÃO APRESENTADA \\ $\mathrm{AO}$, \\ Instituto DE MATEMÁticA E Estatí́stica \\ Universidade DE SÃo PAUlo \\ PARA \\ OBTENÇÃO DO TÍTULO \\ Mestre em Éstatística \\ Programa: Estatística \\ Orientador: Prof. Dr. Fábio Prates Machado
}

Durante o desenvolvimento deste trabalho o autor recebeu auxílio financeiro da $\mathrm{CNPq}$

São Paulo, setembro de 2021 


\section{Uma Introdução ao Processo de Nascimento e Assassinato}

Esta versão da dissertação contém as correções e alterações sugeridas pela Comissão Julgadora durante a defesa da versão original do trabalho, realizada em 26/08/2021. Uma cópia da versão original está disponível no

Instituto de Matemática e Estatística da Universidade de São Paulo.

Comissão Julgadora:

- Prof. Dr. Fábio Prates Machado (orientador) - IME-USP

- Prof. Dr. Alejandro Roldan Correa - UDEA

- Prof. Dr. Carolina Bueno Grejo - Pós-doc IME-USP 


\section{Resumo}

Lima, A. G. Uma Introdução ao Processo de Nascimento e Assassinato. 2021. Dissertação - Instituto de Matemática e Estatística, Universidade de São Paulo, São Paulo, 2021.

Esse texto tem como foco passar pelos resultados apresentados por Aldous e Krebs sobre o Processo de Nascimento e Assassinato, dando atenção nas passagens omitidas por esses autores. Junto a isso, por motivos didáticos, passaremos por alguns resultados importantes do Processo de Ramificação com o intuito de trazer intuição para os resultados postos para o Processo de Nascimento e Assassinato. Com isso posto, apresentaremos o principal resultado a respeito da criticidade do Processo de Nascimento e Assassinato, e faremos então uma demonstração para esse resultado. Seguindo com o texto, iremos então passar a tratar de 4 casos particulas desse resultado, para quando temos a distribuição de assassinato fixadas.

Palavras-chave: Probabilidade, Processos Estocásticos, Partículas Interagentes. 


\section{Abstract}

Lima, A. G. An Introduction to Birth and Assassination Process. 2021. dissertation Mathematics and Statistics Institute, University of São Paulo, São Paulo, 2021.

This text focuses on going through the results presented by Aldous and Krebs about the Birth and Assassination Process, to the passages omitted by these authors. In addition, for didactic reasons, we will go through some important results of the Branching Process in order to bring intuition to the results set for the Birth and Assassination Process. With that said, we will present the main result regarding the criticality of the Birth and Assassination Process, and then we will make a demonstration for this result. Continuing with the text, we will then go on to deal with 4 particular cases of this result, for when we have the killing distribution fixed.

Keywords: Probability, Stochastic Process, Interaction Particles. 


\section{Sumário}

$\begin{array}{ll}\text { Lista de Figuras } & \text { ix }\end{array}$

$\begin{array}{ll}\text { Introdução } & 1\end{array}$

$\begin{array}{ll}\text { Processo de Ramificação } & 3\end{array}$

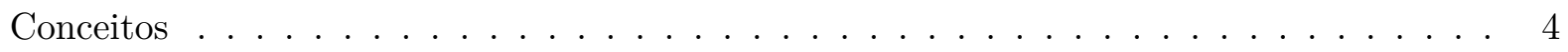

$\begin{array}{ll}\text { Processo de Nascimento e Assassinato } & 9\end{array}$

Casos Particulares $\quad \mathbf{1 5}$

Caso particular - $\phi$ de acordo com a distribuição Exponencial . . . . . . . . . . . . . . 15

Caso particular - $\phi$ de acordo com a distribuição Gama . . . . . . . . . . . . . . . . 15

Caso particular $-\phi$ de acordo com a distribuição Uniforme $(0,1) \ldots \ldots$. . . . . . . 16

Caso particular $-\phi$ de acordo com a distribuição Beta . . . . . . . . . . . . . . . . 16

$\begin{array}{ll}\text { Conclusões } & 19\end{array}$

$\begin{array}{ll}\text { Referências Bibliográficas } & 21\end{array}$ 


\section{Lista de Figuras}

1 Árvore genealógica. . . . . . . . . . . . . . . . . . . . 3

2 Simulação do exemplo. . . . . . . . . . . . . . . . . . . . 5

3 Evolução da sequência $q_{n}$ nos casos que a) $\left.\mathrm{m} \leq 1 \mathrm{e} \mathrm{b}\right) \mathrm{m}>1 \ldots \ldots \ldots$

4 Contrução visual dos conceitos. . . . . . . . . . . . . . . . . . . . . 10

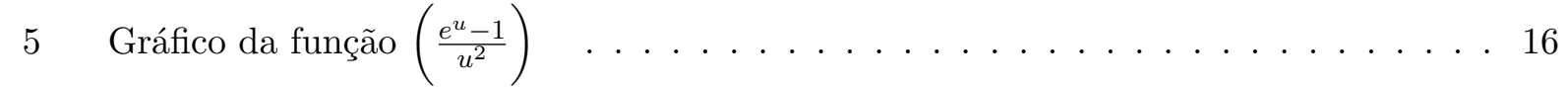

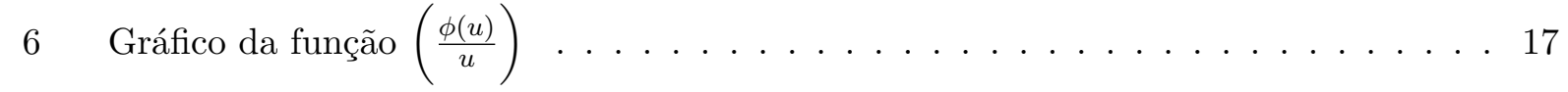




\section{Introdução}

Com o intuito de modelar o desenvolvimento de populações, diversos modelos probabilísticos surgiram. Um dos processos de modelagem mais conhecido é o processo de ramificação, sendo que uma de suas contextualizações mais comuns, é a modelagem de crescimento populacional para verificação da sobrevivência ou não de uma população ao longo de tempo.

Aqui ressaltamos que esse texto possui um foco didático, e como tal, passamos algumas contextualizações desse modelo de forma a torná-lo o mais paupável possível. Primeiro pensando no processo de ramificação a tempo contínuo, podemos pensar o processo como uma modelagem de quantas vezes uma pessoa fará algo até morrer. De fato, para ser mais preciso, na contextualização mais conhecida, pensamos em quantos filhos essa pessoa terá até morrer, sendo que este modelo está definido para qualquer problema onde temos uma processo que acontece repetidas vezes dentro de um intervalo de tempo, onde para cada vez que aconteceu esse evento o processo possa se repetir (filhos tendo outros filhos por exemplo).

O Processo de Nascimento e Assassinato, de Aldous e Krebs [1], que é o processo que estudamos nesse texto, usa como base para sua construção o processo de ramificação à tempo contínuo, impondo no mesmo um comportamento específico em relação ao tempo de vida de cada geração.

O grande objetivo desse texto é entender quais são as condições onde o Processo de Nascimento e Assassinato é um processo supercrítico, isto é, sob quais condições esse processo pode sobreviver indefinidamente com probabilidade positiva, ou subcrítico, ou seja, sob quais condições esse processo morre com probabilidade 1. Essa avaliação é feita tanto sobre esse processo diretamente, como para alguns casos particulares desse processo.

Com isso, o primeiro passo aqui é compreender a fundo os principais resultados para o modelo que estamos usando como base e para tal, iniciamos tratando no primeiro capítulo do processo de ramificação a tempo discreto, onde trazemos um primeiro contato com a área e apresentamos os principais resultados para esse processo, com o intuito de trazer uma intuição para as demonstrações futuras. Já no segundo capítulo seguimos para o Processo de Nascimento e Assassinato, onde trabalhamos praticamente todas as passagens omitidas do texto de Aldous e Krebs. Por fim, no próximo capítulo, tratamos de alguns casos particulares do Processo de Nascimento e Assassinato quando fixamos algumas distribuições.

Já no capítulo de conclusões, comentamos algumas extensões já estudadas para esse processo e citamos uma variação recentemente estudada do Processo de Nascimento e Assassinato para quando o evento de morte acontece de forma mais global. 


\section{Processo de Ramificação}

Seguindo a linha exposta por Schinazi em [2], apresentamos o processo de ramificação sobre a ótica de desenvolvimento populacional. A visualização mais simples disso é olhando para uma árvore genealógica, como vemos abaixo.

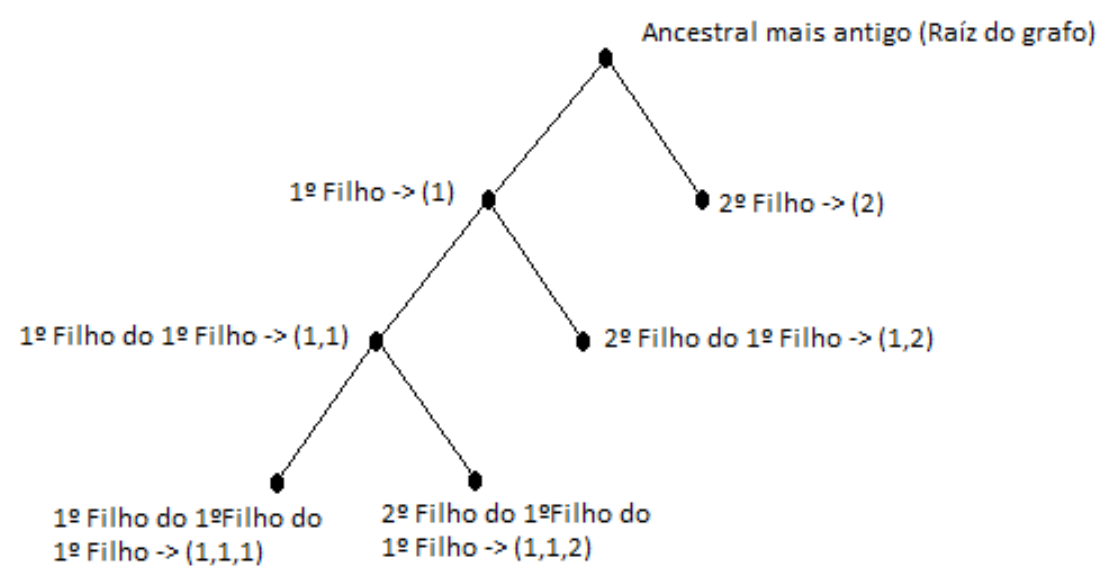

Figura 1: Árvore genealógica. 
De fato, a construção desse processo é pensada da seguinte forma. Temos primeiro um ancestral mais antigo (raíz). Esse ancestral mais antigo terá um número aleatório de descendentes. Seguindo o processo, os filhos também terão cada um um número aleatório de descendentes e por aí o processo segue.

A pergunta que quase sempre gira em torno de processos como esse é, será que esse processo irá sobreviver? Será que conseguimos calcular a probabilidade de em algum momento esse processo deixar de existir? Aqui entrarão as suposições em torno do processo, que nos permitirão responder essas perguntas. Dito isso, vamos então definir esse processo no contexto de probabilidade.

\section{Conceitos}

A primeira suposição que fazemos logo de cara, mas sem causar perda de generalidade, é que temos apenas 1 único vértice raíz, isto é, na geração 0 , antes do processo começar, temos $Z_{0}=1$, onde $Z_{i}$ denota a quantidade de indivíduos $i$-ésima geração do modelo. Isto é, no nosso contexto, $Z_{1}$ representa o número de descendentes originados da raíz, $Z_{2}$ representa o número de descendentes originados dos descendentes originados da raíz e por aí em diante.

Definimos então a sequência de variáveis aleatórias $\left\{Z_{n}\right\}_{n \geq \mathbb{N}}$, que é a definição do processo de ramificação (processo de Bienaymé-Galton-Watson). Para esse processo, temos que o espaço de estados são os Naturais (com o 0 contido) e temos também que o número de descendentes em cada etapa segue uma distribuição de probabilidade discreta não-negativa.

Junto a essa construção, incluímos fim uma última suposição. Durante todo esse trabalho consideramos que o número de descendentes originados em cada vértice se comporta de forma independente aos demais vértices e sobre a mesma distribuição, isto é, dizemos que $N_{i, n}$ e $N_{j, n}$ são independentes e identicamente distribuídos para cada $i \neq j$, onde $i$ e $j$ representam vértices do processo de ramificação na geração $n$ e $N_{i, n}$ representa a variável aleatória associada ao número de descendentes originados do vértice $i$ na geração $n$.

Dessa construção, temos duas principais características para esse modelo. A primeira característica é que o processo descrito acima atende a propriedade Markoviana diretamente.

Definição 1 - (Cadeia De Markov) - Temos que a sequência de variáveis aleatórias $\left\{X_{i}\right\}_{i \in \mathbb{N}}$ é uma cadeia de Markov se, para todo $k \in \mathbb{N}$ temos que

$$
P\left(X_{k}=x \mid X_{k-1}, \ldots, X_{1}\right)=P\left(X_{k}=x \mid X_{k-1}\right)
$$

Em nosso contexto temos diretamente que:

$$
P\left(Z_{k}=n \mid Z_{k-1}, \ldots, Z_{1}\right)=P\left(Z_{k}=n \mid Z_{k-1}\right)
$$

E portanto esse processo é uma cadeia de Markov. Para clarificar um pouco o por que dessa relação ser válida, podemos voltar na definição do processo. Como $Z_{n}$ representa o número de descendentes na geração $n$, e os eventos associados a $N_{i, n}$ são independentes na geração $n$, para saber quantos descendentes teremos na geração $n+1\left(Z_{n+1}\right)$, tudo que precisamos saber é de quantos descendentes temos no processo $\left(Z_{n}\right)$. Aqui notamos a força da suposição que fizemos a pouco sobre $N_{i, n}$.

Notemos que com a propriedade Markoviana, unido a independência dos $N_{i, n}$, conseguimos agora pensar o processo em função de uma distribuição em comum $N$, com mesma distribuição de $N_{i, n}$. Ou seja, quando sabemos $Z_{n-1}$ conseguimos uma relação direta entre o número esperado do tamanho da geração $n\left(E\left[Z_{n} \mid Z_{n-1}=i\right]\right)$ e o número esperado de descendentes originados por cada vértice $(E[N])$. De fato, será sobre essa relação que abordaremos no principal teorema dessa sessão. Antes disso, vamos primeiro olhar um exemplo de como todas essas informações a pouco apresentadas podem ser interligadas.

Exemplo: Seja uma única partícula (ou pessoa) no instante inicial e sejam $N_{i, n}$ tal que $\mathrm{P}\left(N_{i, n}\right.$ $=0)=\frac{1}{4}, \mathrm{P}\left(N_{i, n}=1\right)=\frac{1}{4}$ e $\mathrm{P}\left(N_{i, n}=2\right)=\frac{1}{2}$. Nesse caso, cada vértice da árvore pode gerar de 0 à 2 descendentes, de acordo com as probabilidades definidas. 
Para gerar um simulação dessa construção, podemos realizar o seguinte processo: Suponha uma moeda honesta. Para cada vértice, lance ela para o alto 2 vezes e em cada etapa anote seu resultado. Temos então que o conjunto de possibidades é dado por $\{\{\mathrm{H}, \mathrm{H}\},\{\mathrm{H}, \mathrm{T}\},\{\mathrm{T}, \mathrm{H}\},\{\mathrm{T}, \mathrm{T}\}\}$, onde $\mathrm{H}$ indica cara (head) e $\mathrm{T}$ coroa (tail). Como por suposição a moeda é honesta, temos que a probabildade de cada um dos eventos é $\frac{1}{4}$, e então para simular nosso processo, falaremos que se o resultado desse lançamento for $\{\mathrm{H}, \mathrm{T}\}$ teremos 0 descendentes no vértice i, já caso o resultado seja $\{\mathrm{T}, \mathrm{H}\}$ teremos 1 descendente no vértice i e por fim se o resultado for $\{\mathrm{H}, \mathrm{H}\}$ ou $\{\mathrm{T}, \mathrm{T}\}$ teremos 2 descendentes no vértice i. Podemos então repetir esse processo indefinidamente para cada vértice que ainda não passou por esse processo.

\section{Etapa 1}

-

Nesse momento, foi sorteado $\{\mathrm{H}, \mathrm{H}\}$, e por isso a raíz terá 2 filhos.

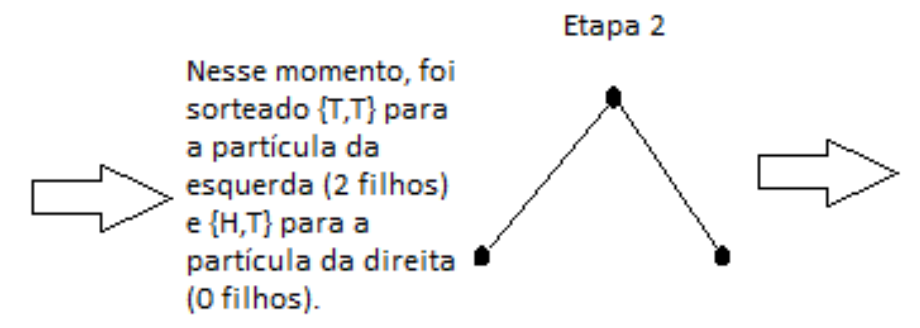

Etapa 4

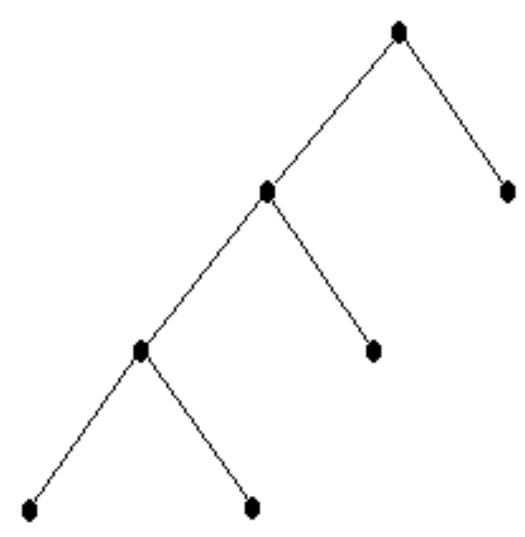

Figura 2: Simulação do exemplo. 
Além disso, vale levantar que para esse exemplo, temos que o número esperado de descendentes de cada vértice é 1,25 , o que veremos futuramente que garante uma probabilidade positiva desse processo existir para sempre. Ora, intuitivamente podemos pensar que se cada pessoa terá em média mais de 1 descendente, a cada geração o número de pessoas vai aumentar e o processo sobrevive. Vamos então formalizar o que sobrevivência, para então apresentar o teorema que liga esses resultados, que pode ser visto em [3].

Chamamos de extinção o evento onde a partir de alguma geração, não temos mais partículas no processo, o que pode ser representado por:

$$
\text { Extinção }:=\left\{\exists k \in \mathbb{N}: Z_{k}=0\right\}
$$

Consequentemente temos que a sobrevivência é a negação da extinção, o que finalmente nos leva ao primeiro teorema que apresentaremos aqui:

Teorema (1). - Seja $\left\{Z_{i}\right\}_{i \in \mathbb{N}}$ um processo de ramificação tal que a distribuição do número de descendentes seja dada por $N$, com $m=E[N]$ e com $P[N=0]+P[N=1]<1$. Então temos que:

i) Se $m \leq 1$ então $P($ Sobrevivência $)=0$. ii) Se $m>1$ então $P$ (Sobrevivência) $>0$.

Como para a demonstração do Teorema usaremos conceitos relativos a função geradora da variável aleatória $Z_{n}$, irei primeiro descrever as propriedades que usaremos.

Primeiro, definiremos as funções geradoras de $Z_{n}$ e $N$ :

$$
\begin{gathered}
G_{n}(s)=E\left[s^{Z_{n}}\right]=\sum_{k \geq 0} s^{k} P\left(Z_{n}=k\right), s \in[-1,1] \\
F(s)=\sum_{k \geq 0} s^{k} P(N=k), s \in[-1,1]
\end{gathered}
$$

Note que $G_{Z_{n}}(0)=P\left(Z_{n}=0\right)$. Além disso, pelo fato de termos fixado que uma única partícula no momento inicial, vale também que na primeira iteração vale $F(s)=G(s)$. Consequentemente, como $Z_{n}$ é uma função aditiva de variáveis independentes, vale que:

$$
G_{n+1}(s)=G\left(G_{n}(s)\right)=G_{n}(G(s))=G_{n}(F(s))=F_{n+1}(s), s \in[-1,1]
$$

Outra propriedade que vale ser levantada é que, para $\mathrm{s} \in(-1,1)$ :

$$
G^{\prime}(s)=\sum_{k \geq 0} k s^{k-1} P(N=k)
$$

E com isso

$$
\lim _{s \rightarrow 1^{-}} G^{\prime}(s)=E[N]=m .
$$

Por fim, a última propriedade que vale ressaltar é que, $G_{n}(s)$ é convexa para s $\in(-1,1)$.

Tendo posto as propriedades que usaremos, podemos partir para a demonstração do Teorema. Demonstração - Nosso interesse aqui é avaliar q $:=\mathrm{P}\left(\right.$ Extinção). Vamos para isso, definir $q_{n}=$ $P\left(Z_{n}=0\right)$.

Note porém, que se $Z_{n}=0$, então $Z_{n+1}=0$, e com isso temos que:

$$
\left\{Z_{1}=0\right\} \subset\left\{Z_{2}=0\right\} \subset \ldots \subset\{\text { Extinção }\} .
$$

E portanto, temos que $q_{1} \leq q_{2} \leq \ldots \leq q$. Mas como

$$
\{\text { Extinção }\}=\bigcup_{n \geq 1}\left\{Z_{n}=0\right\}
$$

E portanto, como $q_{n}$ é crescente e limitado superiormente por 1, temos que: 


$$
\lim _{n \rightarrow \infty} q_{n}=q
$$

Como conseguimos escrever $q_{n}=G_{n}(0)$, então iremos estudar a probabilidade de extinção a partir da função geradora de $Z_{n}$.

Primeiro, notemos que $\mathrm{G}(0)=q_{0}>0$ por suposição. Além disso, temos também que $\mathrm{G}(1)=$ 1 pois $Z_{n}$ é uma distribuição de probabilidade. Tendo isso posto, temos agora que separar em 2 casos, devido as propriedades da função $G$.

i) $\mathrm{m} \leq 1$. Neste caso, como G é convexa, seu gráfico fica acima da diagonal principal (Imagem a seguir) e portanto $\lim _{n \rightarrow \infty} q_{n}=1$ e com isso temos que $\mathrm{q}=1$.
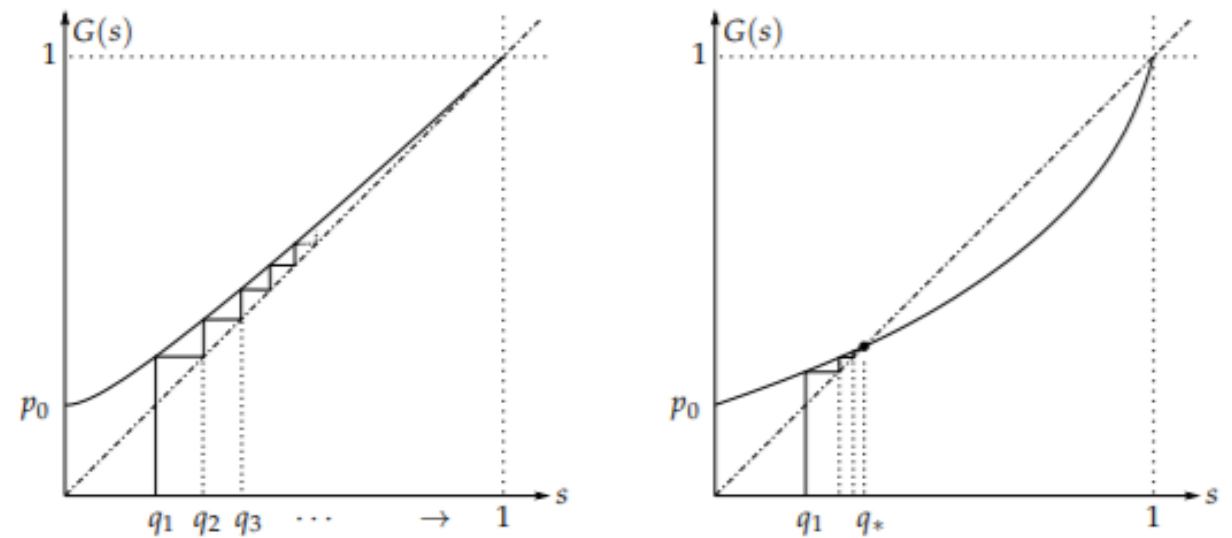

Figura 3: Evolução da sequência $q_{n}$ nos casos que a) $m \leq 1$ e b) $m>1$.

ii) $\mathrm{m}>1$. Neste caso, o gráfico de $\mathrm{G}$ cruza a diagonal principal (Imagem acima) e portanto $\lim _{n \rightarrow \infty} q_{n}=\mathrm{q}^{*}, \operatorname{com~q}^{*}<1$.

Finalizando assim a demonstração do Teorema. De fato, aqui cabe um refino na última passagem da demostração desse teorema, que pode ser encontrada em [2], onde ele detalha melhor o comportamento dessa função em cada um dos casos postos acima.

O resultado enunciado e demonstrado acima, nos permite afirmar se existe uma probabilidade de sobrevivência ou não do processo e servirá como base para o principal resultado desse trabalho. 
Processo de Ramificação 


\section{Processo de Nascimento e Assassinato}

A ideia central dessa pesquisa, surgiu quando Alduos e Krebs desenvolveram uma progressão dos resultados obtidos acima para o Processo de Nascimento e Assassinato [1]. Para compreensão desse resultado, antes precisamos entender a estrutura do Processo de Nascimento e Assassinato.

Diferente do que era feito antes, onde dizíamos que o número descendentes que irão nascer do vértice $i$ segue uma distribuição de probabilidade $N_{i}$, agora nossa preocupação é com o tempo até o nascimento de um novo descendente, ou seja, dizemos agora que o tempo até o nascimento de um novo descendente advindo o vértice $i$, na geração $n$ é uma variável aleatória $B_{i, n}$, onde $B_{i, n}$ está definida em $\mathbb{R}_{+}=\{x \in \mathbb{R}: x>0\}$.

Além disso agora dizemos que o tempo até a morte da partícula presente no vértice $i$ é uma variável aleaória $K_{i}$, com $K_{i}$ definida em $\mathbb{R}_{+}$. Um dos principais diferenciais desse processo para os demais (como o processo de ramificação a tempo contínuo) é que agora dizemos que o 'relógio' de um descendente só inicia quando seu antecessor morre (e por esse motivo estamos omitindo o índice para geração).

Note aqui que essa última diferença é crítica para a construção do processo e diferencia o mesmo fortemente do processo de ramificação a tempo contínuo. Aqui, dado uma partícula, enquanto todos os seus ancestrais não forem extintos, essa partícula sequer está em risco, já para o processo de ramificação tradicional, uma partícula está em risco a partir do momento que nasceu.

Como dito anteriormente, nosso interesse é verificar a sobrevivência ou não do Processo de Nascimento e Assassinato. Para obter tal resultado, precisamos primeiro formalizar o modelo em questão. A primeira formalização (e suposição) que faremos é que $B_{i, n}$ segue uma distribuição de probabilidade Exponencial $(\lambda)$. Essa suposição por si só, nos trás algumas propriedades importantes, como a ausência de memória. Além disso, estamos supondo também que $B_{i, n}$ e $B_{j, n}$ são identicamente distribuídos para todos $i, j$, com $i$ diferente de $j$. Essa suposição também ocorre para vértices em diferentes gerações.

Com todas as suposições feitas, nesse momento iremos iniciar a formalização do processo com o intuito de fazer as demonstrações dos resultados obtidos por Audous e Krebs.

Seja $N$ os inteiros positivos e denote por $N^{f}=\bigcup_{n=0}^{\infty} N^{n}$ o conjunto dos vetores de dimensão n de inteiros positivos $\left(N^{0}=\emptyset\right)$. Para cada $\mathbf{n} \in N^{f}$ (cada possível caminho percorrido), temos que $\mathrm{k}(\mathbf{n})$ representa a dimensão desse vetor $(k(\emptyset)=0)$ e $\sigma(\mathbf{n})=n_{1}+\ldots+n_{k(\mathbf{n})}$ representa a soma do caminho percorrido $(\sigma(\emptyset)=0)$. Abaixo segue a representação visual dos conceitos definidos acima. 


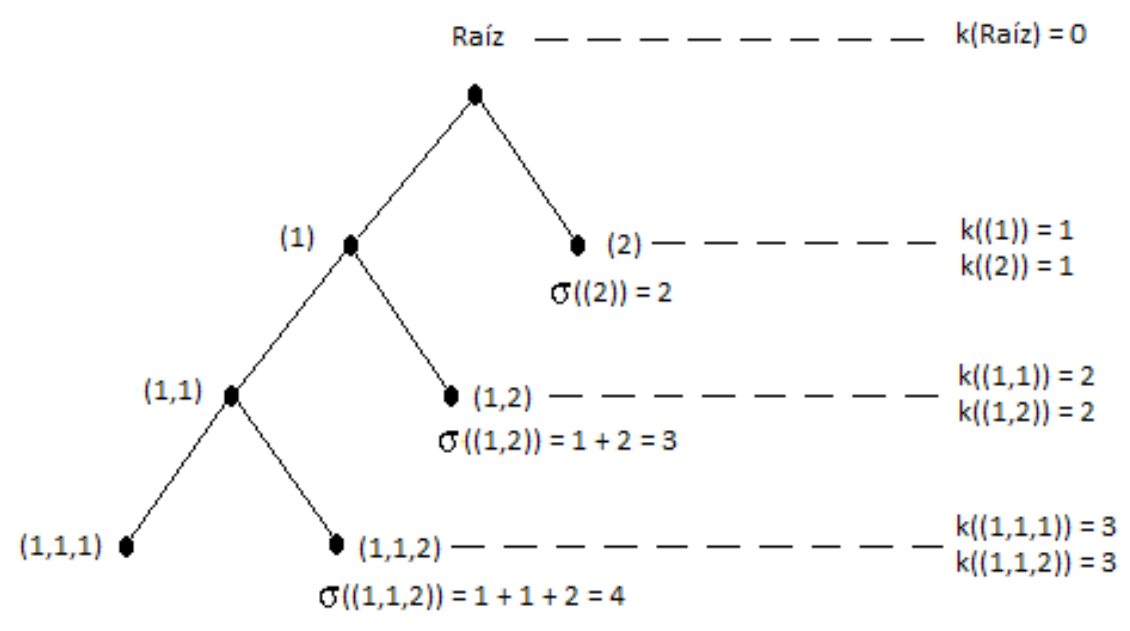

Figura 4: Contrução visual dos conceitos.

Agora fixado $\mathbf{n} \in N^{f}$, definiremos $X_{\mathbf{n}}$ como uma família de processos de Poisson com taxa $\lambda$ (o que temos direto da suposição de exponenciais independentes e com mesma taxa) de tal forma que para todo $\mathbf{n}, \mathbf{m} \in N^{f}, \mathbf{n} \neq \mathbf{m}$, temos que $X_{\mathbf{n}}$ é independente de $X_{\mathbf{m}}$. Essa mesma independência também é válida para $K_{\mathbf{n}}$ e $K_{\mathbf{m}}$.

Note que $X_{\mathbf{n}}$ aqui representa o número de descendentes que o vértice na posição $\mathbf{n}$ terá e $K_{\mathbf{n}}$ faz o papel do tempo de vida até a morte do mesmo vértice a partir do momento que ele se torna em risco. Aqui assumiremos que as famílias de distribuições $\left\{X_{\mathbf{n}}\right\}$ e $\left\{K_{\mathbf{n}}\right\}$ são independentes.

Agora as formalizações feitas, iremos definir o início do processo, que assim como nos processos anteriormente definidos, ocorre em um vértice raíz. Nesse caso, diremos que no instante 0 existe apenas uma partícula, que será indexada por $\emptyset$. Como definido acima, temos que essa partícula produz descendentes de acordo com $X_{\emptyset}$, que entrarão no sistema com os índices $(1),(2)$, ..., de acordo com a ordem do nascimento. Agora, cada partícula nova, digamos $\mathbf{n}$, que entra no sistema começa a produzir descendentes de acordo com $X_{\mathbf{n}}$ e seus descendentes serão indexados por $(\mathbf{n}, 1)$, $(\mathbf{n}, 2), \ldots$, seguindo a ordem de nascimento, formando assim um caminho entre a nova partícula e a raíz.

Um dos pontos que mais diferencia esse processo dos demais, é o fato de que uma partícula só se torna exposta quando seu antecessor direto morre, ou seja, estamos definindo $Y_{i}$ como uma variável aleatória correspondente ao tempo total passado até a morte na partícula $i$. Com isso, temos que no momento inicial a partícula raíz está em risco desde o momento 0 e continuará a produzir descendentes até $Y_{\emptyset}=K_{\emptyset}$, quando será removida do sistema. Generalizando, temos que para $\mathbf{n}=\left(n_{1}, \ldots, n_{k-1}, n_{k}\right)$ e $\mathbf{n}^{\prime}=\left(n_{1}, \ldots, n_{k-1}\right), \mathrm{k}>0$, vale que $Y_{\mathbf{n}}=Y_{\mathbf{n}^{\prime}}+K_{\mathbf{n}}$.

Definido todo o processo, iremos estudar agora sobre quais condições esse processo morre com probabilidade 1, isto é, existe algum $\mathrm{t}<\infty$ tal que não exista nenhuma partícula no sistema no momento t? Para responder essa pergunta, Aldous e Kreb elaboraram o seguinte Teorema:

Teorema (2). - Seja $\mathbb{B}$ um Processo de Nascimento e Assassinato com taxa de nascimento $\lambda$ e tal que a distribuição de morte tenha uma função geradora de momentos $\phi$, com $\phi$ finita em alguma vizinhança do 0. Temos então que:

I) Se $\min _{u>0} \lambda u^{-1} \phi(u)<1$, então o processo morrerá em algum momento com probabilidade 1 .

II) Caso $\min _{u>0} \lambda u^{-1} \phi(u)>1$, então a probabilidade do processo sobreviver indefinidamente é 
maior do que 0 .

Observação: Note que este resultado é uma progressão natural dos resultados obtidos anteriormente, agora adaptado ao novo processo.

Demonstração Parte I: Sejam $\left\{B_{v_{i}}\right\}, \mathrm{v}, \mathrm{i}=1,2, \ldots$ variáveis aleatórias exponenciais independentes com parâmetro $\lambda$ e $\left\{K_{i}\right\}$ variáveis aleatórias independentes com função de distribuição $\mathrm{F}$ e função geradora de momentos $\phi(u)$.

Temos que $\mathbb{B}$ morre com probabilidade 1 se o número esperado de partículas no sistema é finito, isto é

$$
\mathbb{E}[\text { número de partículas criadas }]=\sum_{\mathbf{n}} \mathbb{P}(\mathbf{n} \text { nascer })<\infty
$$

Note que para $\mathbf{n}=\left(n_{1}, \ldots, n_{k-1}, n_{k}\right)$ nascer, temos que seu ancestral na geração $i$ teve pelo menos $n_{i}$ descendentes, para cada $i$ menor do que $k$. Nessa demonstração, $i$ fará o papel de mapear a geração e, fixado um $i, v$ fará o papel de mapear os descendentes $1, \ldots, n_{i}$. Com isso, podemos escrever a equação de cima da seguinte forma:

$$
\mathbb{P}(\mathbf{n} \text { nascer })=\mathbb{P}\left[\sum_{i=1}^{j} \sum_{v=1}^{n_{i}} B_{v_{i}}<\sum_{i=1}^{j} K_{i}, \quad \mathrm{j}=1, \ldots, \mathrm{k}(\mathbf{n})\right]
$$

Como o evento $\left\{\sum_{i=1}^{j} \sum_{v=1}^{n_{i}} B_{v_{i}}<\sum_{i=1}^{j} K_{i}, \quad \mathrm{j}=1, \ldots, \mathrm{k}(\mathbf{n})\right\}$ está contido no evento onde olhamos somente para $\left\{\sum_{i=1}^{k(\mathbf{n})} \sum_{v=1}^{n_{i}} B_{v_{i}}<\sum_{i=1}^{k(\mathbf{n})} K_{i}\right\}$, temos então que:

$$
\mathbb{P}\left[\sum_{i=1}^{j} \sum_{v=1}^{n_{i}} B_{v_{i}}<\sum_{i=1}^{j} K_{i}, \quad \mathrm{j}=1, \ldots, \mathrm{k}(\mathbf{n})\right] \leq \mathbb{P}\left[\sum_{i=1}^{k(\mathbf{n})} \sum_{v=1}^{n_{i}} B_{v_{i}}<\sum_{i=1}^{k(\mathbf{n})} K_{i}\right]
$$

Logo

$$
\mathbb{P}(\mathbf{n} \text { nascer }) \leq \mathbb{P}\left[\sum_{i=1}^{k(\mathbf{n})} \sum_{v=1}^{n_{i}} B_{v_{i}}<\sum_{i=1}^{k(\mathbf{n})} K_{i}\right]
$$

Então, unindo (1) e (2) temos que

$$
\begin{gathered}
\sum_{\mathbf{n}} \mathbb{P}\left[\sum_{i=1}^{k(\mathbf{n})} \sum_{v=1}^{n_{i}} B_{v_{i}}<\sum_{i=1}^{k(\mathbf{n})} K_{i}\right]=\sum_{\mathbf{n}} \mathbb{P}\left[\sum_{i=1}^{k(\mathbf{n})} K_{i}-\sum_{i=1}^{k(\mathbf{n})} \sum_{v=1}^{n_{i}} B_{v_{i}}>0\right] \\
=\sum_{\mathbf{n}} \mathbb{P}\left[u\left(\sum_{i=1}^{k(\mathbf{n})} K_{i}-\sum_{i=1}^{k(\mathbf{n})} \sum_{v=1}^{n_{i}} B_{v_{i}}\right)>0\right](u>0) \\
=\sum_{\mathbf{n}} \mathbb{P}\left[\exp \left\{u\left(\sum_{i=1}^{k(\mathbf{n})} K_{i}-\sum_{i=1}^{k(\mathbf{n})} \sum_{v=1}^{n_{i}} B_{v_{i}}\right)\right\}>1\right](u>0) \\
\leq \sum_{\mathbf{n}} \mathbb{E}\left[\exp \left\{u\left(\sum_{i=1}^{k(\mathbf{n})} K_{i}-\sum_{i=1}^{k(\mathbf{n})} \sum_{v=1}^{n_{i}} B_{v_{i}}\right)\right\}\right](u>0)
\end{gathered}
$$

Sendo que a última desigualdade se da pela desigualdade de Markov. Agora, por (3), temos agora que

$$
\sum_{\mathbf{n}} \mathbb{P}\left[\sum_{i=1}^{k(\mathbf{n})} \sum_{v=1}^{n_{i}} B_{v_{i}}<\sum_{i=1}^{k(\mathbf{n})} K_{i}\right] \leq \sum_{\mathbf{n}} \mathbb{E}\left[\exp \left\{u\left(\sum_{i=1}^{k(\mathbf{n})} K_{i}-\sum_{i=1}^{k(\mathbf{n})} \sum_{v=1}^{n_{i}} B_{v_{i}}\right)\right\}\right](u>0)
$$




$$
=\sum_{\mathbf{n}}\left(\prod_{i=1}^{k(\mathbf{n})} \mathbb{E}\left[\exp \left(u K_{i}\right)\right] \prod_{i=1}^{k(\mathbf{n})} \prod_{v=1}^{n_{i}} \mathbb{E}\left[\exp \left(-u B_{v_{i}}\right)\right]\right)
$$

Onde essa passagem se dá devido a independência de $\left\{B_{i}\right\}$ e $\left\{K_{i}\right\}$. Além disso, como $\left\{B_{i}\right\}$ segue uma Exponencial $(\lambda)$, temos então que $\mathbb{E}\left[\exp \left(-u B_{v_{i}}\right)\right]=\frac{\lambda}{u+\lambda}$, bem como $\mathbb{E}\left[\exp \left(u K_{i}\right)\right]=\phi(u)$. Logo,

$$
\begin{gathered}
\sum_{\mathbf{n}}\left(\prod_{i=1}^{k(\mathbf{n})} \mathbb{E}\left[\exp \left(u K_{i}\right)\right] \prod_{i=1}^{k(\mathbf{n})} \prod_{v=1}^{n_{i}} \mathbb{E}\left[\exp \left(-u B_{v_{i}}\right)\right]\right) \\
=\sum_{\mathbf{n}}\left(\prod_{i=1}^{k(\mathbf{n})} \phi(u) \prod_{i=1}^{k(\mathbf{n})} \prod_{v=1}^{n_{i}} \frac{\lambda}{u+\lambda}\right)
\end{gathered}
$$

Temos agora então que dado um $\mathrm{k}(\mathbf{n})=\mathrm{k}$, vale que

$$
\begin{gathered}
\left(\prod_{i=1}^{k(\mathbf{n})} \phi(u) \prod_{i=1}^{k(\mathbf{n})} \prod_{v=1}^{n_{i}} \frac{\lambda}{u+\lambda}\right) \\
=\left(\phi(u)^{k} \sum_{\mathbf{n}: k(\mathbf{n})=k}\left(\frac{\lambda}{u+\lambda}\right)^{n_{1}+\ldots+n_{k(\mathbf{n})}}\right) \\
=\left(\phi(u)^{k} \sum_{\mathbf{n}: k(\mathbf{n})=k}\left(\frac{\lambda}{u+\lambda}\right)^{\sigma(\mathbf{n})}\right)
\end{gathered}
$$

Agora unindo (5) com (6), temos que

$$
\sum_{\mathbf{n}} \mathbb{P}\left[\sum_{i=1}^{k(\mathbf{n})} \sum_{v=1}^{n_{i}} B_{v_{i}}<\sum_{i=1}^{k(\mathbf{n})} K_{i}\right] \leq \sum_{k=1}^{\infty}\left(\phi(u)^{k} \sum_{\mathbf{n}: k(\mathbf{n})=k}\left(\frac{\lambda}{u+\lambda}\right)^{\sigma(\mathbf{n})}\right)
$$

Com isso, temos que

$$
\mathbb{E}[\text { número de partículas criadas }] \leq \sum_{k=1}^{\infty}\left(\phi(u)^{k} \sum_{\mathbf{n}: k(\mathbf{n})=k}\left(\frac{\lambda}{u+\lambda}\right)^{\sigma(\mathbf{n})}\right)
$$

Note aqui que ainda estamos somando em $\mathbf{n}: k(\mathbf{n})=k$. Como nosso interesse é seguir somando em $k$, vamos então mudar o intervalor de soma da segunda parte da equação. Para um $k$ fixado, temos $\left(\begin{array}{c}m-1 \\ k-1\end{array}\right)$ formas de somar um total de $m$, e por isso, vale que:

$$
\sum_{\mathbf{n}: k(\mathbf{n})=k}\left(\frac{\lambda}{u+\lambda}\right)^{\sigma(\mathbf{n})}=\sum_{m=k}^{\infty}\left(\begin{array}{c}
m-1 \\
k-1
\end{array}\right)\left(\frac{\lambda}{u+\lambda}\right)^{m}
$$

Consequentemente por (8) e (9) temos que

$$
\mathbb{E}\left[\mathrm{N}^{\mathrm{o}} \text { part. criadas }\right] \leq \sum_{k=1}^{\infty}\left(\phi(u)^{k} \sum_{m=k}^{\infty}\left(\begin{array}{c}
m-1 \\
k-1
\end{array}\right)\left(\frac{\lambda}{u+\lambda}\right)^{m}\right)
$$

O que, desenvolvendo as contas e completando uma Hipergeométrica nos dá que

$$
\mathbb{E}\left[\mathrm{N}^{\mathrm{o}} \text { part. criadas }\right] \leq \sum_{k=1}^{\infty}\left(\phi(u)^{k}\left(\frac{\lambda}{u}\right)^{k} \sum_{m=k}^{\infty}\left(\begin{array}{c}
m-1 \\
k-1
\end{array}\right)\left(\frac{\lambda}{u+\lambda}\right)^{m-k}\left(\frac{u}{u+\lambda}\right)^{k}\right)
$$

Logo

$$
\mathbb{E}[\text { número de partículas criadas }] \leq \sum_{k=1}^{\infty}\left(\phi(u)^{k}\left(\frac{\lambda}{u}\right)^{k}\right)
$$


Como a série converge se $\min _{u>0} \lambda u^{-1} \phi(u)<1$, então o número esperado de partículas no sistema é finito, o que implica que o processo morrerá, concluindo a primeira parte da demonstração. Demonstração Parte II: Antes de iniciar a prova da segunda parte do teorema, irei primeiro provar dois resultados intermediários.

Lema (1). - Sejam $X_{1}, X_{2}, \ldots$ variáveis aleatórias independentes e identicamente distribuídas com $\mathbb{E}\left[X_{1}\right]<0$ e $P\left(X_{1}>0\right)>0$. Seja $\phi(u)=\mathbb{E}\left[e^{u X_{1}}\right]$ finita em alguma vizinhança de 0. Seja $\rho=$ $\min _{u>0} \phi(u)$, então:

$$
\lim _{n \rightarrow \infty} n^{-1} \log P\left(\sum_{1}^{k} X_{j}>0, k=1, \ldots, n\right)=\log \rho
$$

$[1]$

Demonstração do Lema 1: Pelo teorema de grandes deviações de Chernoff [4] temos esse limite para $n^{-1} \log \mathrm{P}\left[\sum_{1}^{k} X_{j}>0\right]$.

Além disso, temos que:

$$
1>P\left(\sum_{1}^{k} X_{j}>0, k=1, \ldots, n \mid \sum_{1}^{n} X_{j}>0\right)>\frac{1}{n}
$$

Onde o resultado acima é obtido pensando no passeio aleatório iniciando em $(0,1)$ e nunca cruzando o ponto $(\mathrm{x}, 0)$ até que no momento $n$ ele seja positivo. Consequentemente

$$
\begin{gathered}
1>\frac{P\left[\sum_{1}^{k} X_{j}>0, k=1, \ldots, n\right]}{P\left[\sum_{1}^{n} X_{j}>0\right]}>\frac{1}{n} \\
P\left[\sum_{1}^{n} X_{j}>0\right]>P\left[\sum_{1}^{k} X_{j}>0, k=1, \ldots, n\right]>\frac{1}{n} P\left[\sum_{1}^{n} X_{j}>0\right] \\
\log P\left[\sum_{1}^{n} X_{j}>0\right]>\log P\left[\sum_{1}^{k} X_{j}>0, k=1, \ldots, n\right]>\log \left(\frac{1}{n} P\left[\sum_{1}^{n} X_{j}>0\right]\right) \\
\log P\left[\sum_{1}^{n} X_{j}>0\right]>\log P\left[\sum_{1}^{k} X_{j}>0, k=1, \ldots, n\right]>\log \left(\frac{1}{n} P\left[\sum_{1}^{n} X_{j}>0\right]\right)
\end{gathered}
$$

Dividindo tudo por $\mathrm{n}$ a aplicando o limite, temos que o lado esquerdo da equação se encaixa no teorema de Chernoff.

$$
\log \rho=\lim _{n \rightarrow \infty} n^{-1} \log P\left(\sum_{1}^{k} X_{j}>0\right)>\lim _{n \rightarrow \infty} n^{-1} \log \frac{1}{n} P\left(\sum_{1}^{k} X_{j}>0, k=1, \ldots, n\right)
$$

Como

$$
\lim _{n \rightarrow \infty} n^{-1} \log \frac{1}{n} P\left(\sum_{1}^{k} X_{j}>0\right)=\lim _{n \rightarrow \infty} n^{-1} \log \frac{1}{n} P\left(\sum_{1}^{k} X_{j}>0\right)-\frac{\log (n)}{n}=\log \rho
$$

Temos então a prova do Lema 1 .

Lema (2). - Se $\min _{u>0} \lambda u^{-1} \phi(u)>1$, então existe $k$ tal que $\sum_{k(\mathbf{n})=k} P[\mathbf{n}$ nascer $]>1$.

Demonstração do Lema 2: Notemos primeiro que ao falar que $\sum_{k(\mathbf{n})=k} \mathrm{P}[\mathbf{n}$ nascer $]>1$, estamos falando que em pelo menos uma das gerações temos que ter o número esperado de partículas nascidas nessa geração maior do que 1. Esse resultado conversa diretamente com os resultados que temos dos outros modelos de árvores já estudados aqui nesse texto. Partindo agora para a demonstração de fato, temos o seguinte. 
Sejam $N_{1}, N_{2}, \ldots$ variáveis aleatórias independentes com distribuição comum geométrica, isto é, $\mathrm{P}[\mathrm{N}=\mathrm{j}]=p q^{j-1}, \mathrm{j}=1,2, \ldots$, onde $p>0$ e $q=1-p$. Suponha $N_{1}, N_{2}, \ldots,\left\{B_{v_{j}}\right\}$ e $\left\{K_{j}\right\}$ mutualmente independentes. Para $\mathrm{n}=1,2, \ldots$ definimos $Z_{n}=K_{n}-\sum_{v=1}^{N_{n}} B_{v_{n}}$. Note aqui que $\left\{Z_{n}\right\}$ são i.i.d. Além disso, temos que:

$$
\mathbb{E}\left[e^{u Z_{n}}\right]=\mathbb{E}\left(e^{u K_{n}}\right) \mathbb{E}\left(e^{\left.-u \sum_{v=1}^{N_{n}} B_{v_{n}}\right\}}\right)=\phi(u) \frac{\lambda p}{(\lambda p+u)}
$$

Condicionando em $\left(N_{1}, \ldots, N_{k}\right)=\left(n_{1}, \ldots, n_{k}\right)$ temos que

$$
P\left[\sum_{i}^{j} Z_{i}>0, j=1, \ldots, k \mid\left(N_{1}, \ldots, N_{k}\right)=\left(n_{1}, \ldots, n_{k}\right)\right]=\mathbb{P}\left[\sum_{i=1}^{j} \sum_{v=1}^{n_{i}} B_{v_{i}}<\sum_{i=1}^{j} K_{i}, \quad \mathrm{j}=1, \ldots, \mathrm{k}\right]
$$

Multiplicando ambos os lados por $\mathrm{P}\left[\left(N_{1}, \ldots, N_{k}\right)=\left(n_{1}, \ldots, n_{k}\right)\right]$ e somando sobre todo $\mathbf{n}$ tal que $\mathrm{k}(\mathbf{n})=k$, temos que:

$$
\begin{aligned}
P\left[\sum_{i}^{j} Z_{i}>0, j=1, \ldots, k\right] & =\sum_{k(\mathbf{n})=k} \mathbb{P}\left[\sum_{i=1}^{j} \sum_{v=1}^{n_{i}} B_{v_{i}}<\sum_{i=1}^{j} K_{i}, \quad \mathrm{j}=1, \ldots, \mathrm{k}\right] p^{k} q^{n_{1}+\ldots+n_{k}} \\
& =p^{k} \sum_{k(\mathbf{n})=k} \mathbb{P}(\mathbf{n} \text { nascer }) q^{\sigma(\mathbf{n})}
\end{aligned}
$$

Supondo $\min _{u>0} \lambda u^{-1} \phi(u)=1+\epsilon$, podemos escolher $p$ suficientemente pequeno tal que:

$$
\begin{gathered}
\mathbb{E}\left[e^{u Z_{n}}\right]=\phi(u) \frac{\lambda p}{(\lambda p+u)} \\
\min _{u>0} \mathbb{E}\left[e^{u Z_{n}}\right]=\min _{u>0} \phi(u) \frac{\lambda p}{(\lambda p+u)} \\
p^{-1} \min _{u>0} \mathbb{E}\left[e^{u Z_{n}}\right]=\min _{u>0} \phi(u) \frac{\lambda}{(\lambda p+u)}>1+\frac{1}{2} \epsilon
\end{gathered}
$$

Aplicando o Lema 1 para $\left\{Z_{n}\right\}$, e supondo $k$ suficientemente grande, temos que:

$$
p^{k} \sum_{k(\mathbf{n})=k} \mathbb{P}(\mathbf{n} \text { nascer }) q^{\sigma(\mathbf{n})}=P\left[\sum_{i}^{j} Z_{i}>0, j=1, \ldots, k\right]>p^{k}\left(1+\frac{1}{2} \epsilon\right)^{k} .
$$

Dividindo os dois lados da desigualdade por $p^{k}$ e jogando $p \rightarrow 0$ (isto é, $q \rightarrow 1$ ), temos que:

$$
\sum_{k(\mathbf{n})=k} \mathbb{P}(\mathbf{n} \text { nascer })>\left(1+\frac{1}{2} \epsilon\right)^{k}>1
$$

O que finaliza a demonstração do Lema 2.

Agora iremos retornar a demonstração da segunda parte do Teorema. Para tal, definiremos um processo especial. Diremos que uma partícula está em um processo especial se é a partícula ancestral ou se a partícula da geração $n k$ é descendente de uma partícula especial da geração $(n-1) k$, sem ancestrais vivos. Mas como o número esperado de partículas na geração $n k$ é maior que 1 , o processo de partículas especiais é supercrítico.

Mas como esse processo de partículas especiais é supercrítico, quando acoplamos esse processo com o Processo de Nascimento e Assassinato, temos que esse processo também é supercrítico, o que encerra a demonstração do Teorema. 


\section{Casos Particulares}

\section{Caso particular - $\phi$ de acordo com a distribuição Exponencial}

Com o intuito de apresentar algo mais paupável, nessa seção iremos trabalhar o Processo de Nascimento e Assassinato sobre o caso particular onde supomos que a morte segue uma distribuição exponencial, bem como o nascimento. Isto é, agora dizemos que $K_{i}$ tem distribuição exponencial com taxa $\mu$ e $\mathbb{E}\left[\exp \left(u K_{i}\right)\right]=\phi(u)=\frac{\mu}{\mu-u}$. Com essa nova suposição imposta, temos agora que:

Corolário (1). : Seja $\mathbb{B}$ um Processo de Nascimento e Assassinato com taxa de nascimento $\lambda e$ tal que a distribuição de morte seja uma exponencial com taxa $\mu$. Temos então que: I) Se $\mu>$ $4 \lambda$, então o processo morrerá em algum momento com probabilidade 1. II) Caso $\mu<4 \lambda$, então a probabilidade do processo sobreviver indefinidamente é maior do que 0.

Demonstração: Segue do Teorema de Aldous e Krebs uma vez que $\phi(u)=\frac{\mu}{\mu-u}$ e verificando que $\min _{0<u<\mu} \frac{\lambda}{u} \frac{\mu}{\mu-u}<1 \Leftrightarrow \mu>4 \lambda$ e $\min _{0<u<\mu} \frac{\lambda}{u} \frac{\mu}{\mu-u}>1 \Leftrightarrow \mu<4 \lambda$, o que finaliza a demonstração.

\section{Caso particular - $\phi$ de acordo com a distribuição Gama}

Seguindo com as extenções para casos particulares, trabalharemos agora sobre a distribuição $\operatorname{Gama}(\alpha, \beta)$. Aqui temos agora que $K_{i}$ segue uma distribuição $\operatorname{Gama}(\alpha, \beta)$ e com isso temos que:

$$
\mathbb{E}\left[\exp \left(u K_{i}\right)\right]=\phi(u)=\left(\frac{\beta}{\beta-u}\right)^{\alpha}
$$

Nosso problema agora se resume em aplicar o Teorema de Aldous e Krebs diretamente, isto é

$$
\min _{\beta>u>0}\left(\frac{\beta}{\beta-u}\right)^{\alpha} \frac{\lambda}{u}<1<=>\min _{\beta>u>0} \frac{\lambda \beta^{\alpha}}{u(\beta-u)^{\alpha}}<1
$$

Ora, minimizar essa função em relação a $u$, deve ser uma tarefa simples, uma vez que aqui basta maximizar o denominador da função para esses parâmetros. Aqui, vale notar um detalhe, por exigência do Teorema de Aldous e Krebs, $\phi$ precisa ser finita em alguma vizinhança do 0 , e por isso a necessidade de restringir o intervalo de $u$ para $(0, \beta)$, e consequentemente, dentro desse intervalo vale que

$$
\min _{\beta>u>0} \frac{\lambda \beta^{\alpha}}{u(\beta-u)^{\alpha}}<1 \Leftrightarrow \frac{\lambda \beta^{\alpha}}{\left(\frac{\beta}{\alpha+1}\right)\left(\beta-\left(\frac{\beta}{\alpha+1}\right)\right)^{\alpha}}<1
$$

Após algumas manipulações algébricas, obtemos aqui que

$$
\frac{\lambda \beta^{\alpha}}{\left(\frac{\beta}{\alpha+1}\right)\left(\beta-\left(\frac{\beta}{\alpha+1}\right)\right)^{\alpha}}<1 \Leftrightarrow \frac{\lambda(\alpha+1)^{(\alpha+1)}}{\beta \alpha^{\alpha}}<1
$$

Consequentemente, temos que o processo é subcrítico se $\lambda<\frac{\beta \alpha^{\alpha}}{(\alpha+1)^{(\alpha+1)}}$ e supercrítico se $\lambda>\frac{\beta \alpha^{\alpha}}{(\alpha+1)^{(\alpha+1)}}$. 


\section{Caso particular - $\phi$ de acordo com a distribuição Uniforme $(0,1)$}

Agora para o caso onde temos que $K_{i}$ segue uma distribuição Uniforme $(0,1)$ e com isso temos que

$$
\mathbb{E}\left[\exp \left(u K_{i}\right)\right]=\phi(u)=\frac{e^{u}-1}{u}
$$

Com isso, temos que achar a região de convergência para $\sum_{k=1}^{\infty}\left(\phi(u)^{k}\left(\frac{\lambda}{u}\right)^{k}\right)$ se resume em achar o conjunto onde

$$
\min _{u>0}\left(\frac{e^{u}-1}{u}\right) \frac{\lambda}{u}<1
$$

Trabalhando isoladamente a parte $\left(\frac{e^{u}-1}{u^{2}}\right)$ da função acima, temos que existe um ponto de mínimo para essa função em u, como podemos ver no gráfico abaixo

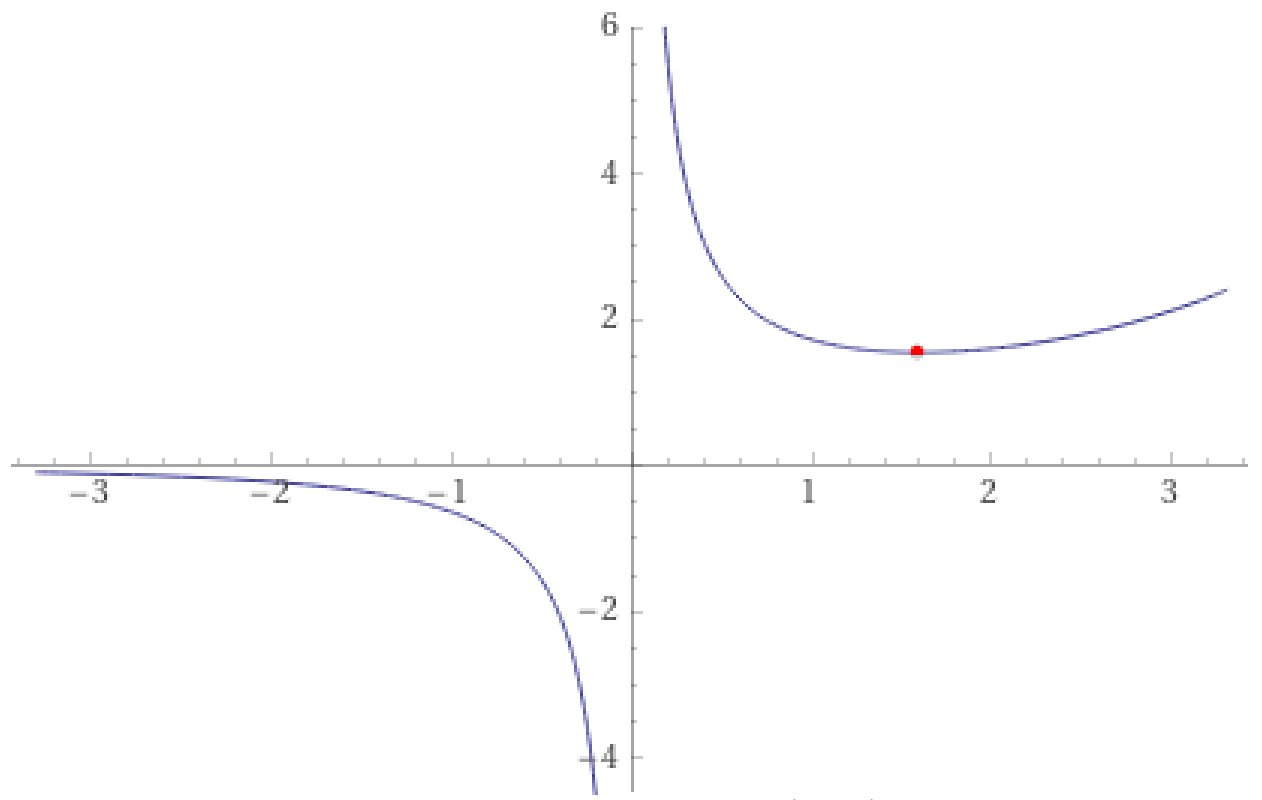

Figura 5: Gráfico da função $\left(\frac{e^{u}-1}{u^{2}}\right)$

E como temos que $\min _{u>0}\left(\frac{e^{u}-1}{u^{2}}\right)$ possui mínimo nesse conjunto, então a região de convergência para o processo é dado em $\lambda<\frac{1}{C}$, onde C é o mínimo da função descrita acima, e que por simulação temos que $\mathrm{C} \approx 1.544143$.

\section{Caso particular - $\phi$ de acordo com a distribuição Beta}

Agora para o caso onde temos que $K_{i}$ segue uma distribuição $\operatorname{Beta}(\alpha, \beta)$ e com isso temos que:

$$
\mathbb{E}\left[\exp \left(u K_{i}\right)\right]=\phi(u)=1+\sum_{k=1}^{\infty}\left(\prod_{r=0}^{k-1} \frac{\alpha+r}{\alpha+\beta+r}\right) \frac{u^{k}}{k !}
$$

Com isso, temos que:

$$
\min _{u>0} \lambda u^{-1} \phi(u)=\min _{u>0} \lambda u^{-1}\left(1+\sum_{k=1}^{\infty}\left(\prod_{r=0}^{k-1} \frac{\alpha+r}{\alpha+\beta+r}\right) \frac{u^{k}}{k !}\right)
$$




$$
\leq \min _{u>0} \lambda u^{-1}\left(1+\sum_{k=1}^{\infty} \frac{u^{k}}{k !}\right)=\min _{u>0} \lambda u^{-1} e^{u}=\lambda e
$$

E com o resultado acima temos uma cota superior para $\lambda$ de forma que o processo possua uma probabilidade positiva de sobrevivência.

Apesar disso, vemos que essa cota não é muito precisa dado a desigualdade usada. Para atacar esse problema, obteremos o ponto de mínimo para $\alpha$ e $\beta$ fixados. Para $\alpha=1$ e $\beta=1$, temos o caso da uniforme, onde nosso simulador apresentou o mesmo resultado, $\mathrm{C} \approx 1.544143$. Para o caso onde $\alpha=2$ e $\beta=2$, nosso simulador apresentou $\mathrm{C} \approx 1.478268$. Para o caso onde $\alpha=1$ e $\beta=2$, nosso simulador apresentou $\mathrm{C} \approx 1.093724$. Seguem os gráficos para esses 3 casos abaixo:

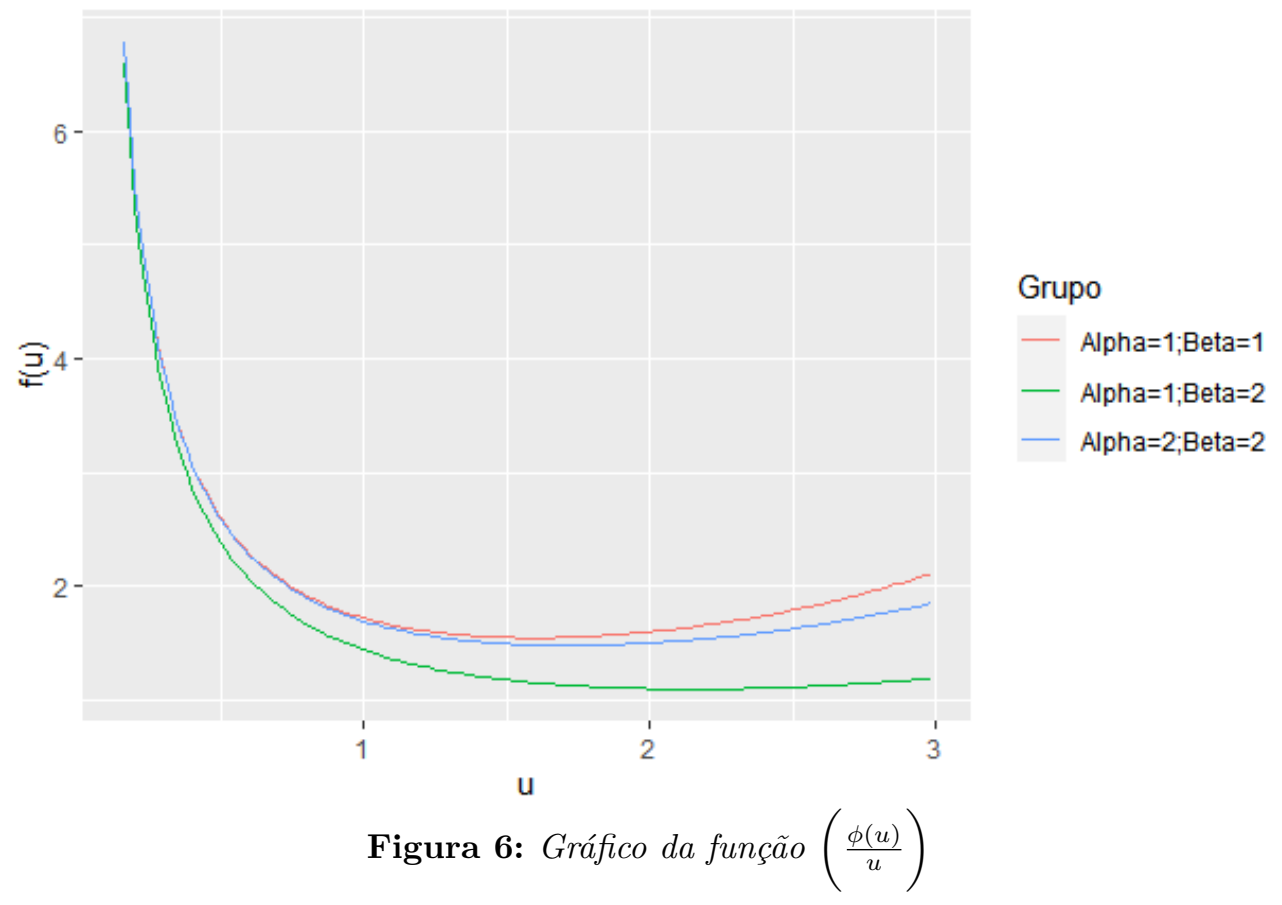

Com isso, temos a intuição de que existe um ponto de convergência para o processo para todos $\alpha$ e $\beta$ fixados, possibilitando assim expandir o mapa de $\alpha$ e $\beta$ em busca de um $\mathrm{C}$ interpolado por esses parâmetros. 


\section{Conclusões}

O texto de Aldous e Krebs possui uma brilhança gigante ao tratar de um processo tão complexo em apenas 4 páginas. Essa capacidade de sintetizar tantas informações em tão poucas linhas salta aos olhos e abre as portas para estudos diversos sobre o Processo de Nascimento e Assassinato, bem como esse texto.

Apesar disso, algumas perguntas permanecem sobre esse processo, como por exemplo, a pergunta de o que acontece no ponto crítico $\min _{u>0} \lambda u^{-1} \phi(u)=1$. Essa pergunta já foi estudada para o caso particular de quando a distribuição de morte é uma Exponancial( $\mu$ ), que foi respondida por Bordenave em [5], onde é demonstrado que esse processo é subcrítico quando $\mu=4 \lambda$.

Ainda a procura da resposta acima, também já foi estudado o resultado sobre o ponto crítico para distribuições mais gerais por Kortchemski em [6], onde é demonstrado a extinção do Processo de Nascimento e Assassinato sob o ponto crítico.

E como dito anteriormente, esse texto de Aldous e Krebs abriu a porta para diversos estudos sobre o que acontece no caso crítico do processo, mas essa inspiração não parou por aí. Esse texto também nos levanta perguntas de como processos similares, mas com algumas diferenças na dinâmica no processo de morte se comportam. Essa pergunta foi abordada por C. Grejo, F. Lopes, F. Machado, A. Roldán-Correa em [7], onde é estudado o processo quando o relógio de toda a geração é o mesmo, isto é, todas as partículas de uma geração morrem ao mesmo momento. 


\section{Referências Bibliográficas}

[1] David Aldous and William B.Krebs, The 'birth-and-assassination' process, Statistics Probability Letters, vol 10, 427-430, 1990.

[2] Rinaldo Schinazi, Classical and spatial stochastic processes, Birkhäuser Boston, 1999.

[3] Rabi N. Bhattacharya, Edward C. Waymire, Stochastic Processes with Applications, Classics in Applied Mathematics, 61, Wiley, 1990.

[4] Sheldon M. Ross, Stochastic processes, Wiley, 1983.

[5] C. Bordenave, On the birth-and-assassination process, with an application to scotching a rumor in a network, Elect. J. Probab., 13, 2014-2030, 2008.

[6] I. Kontchemski, Predator-prey dynamics on infinite trees: a branching random walk approach, J. Theoret. Probab., 29, 1027-1046, 2016.

[7] C. Grejo, F. Lopes, F. Machado, A. Roldán-Correa, Evolution with mass extinction on $\mathbb{T}_{d}^{+}$, arXiv:1710.11150. 